\title{
THE ROLE OF INTRAOCULAR LENS EXCHANGE IN THE MANAGEMENT OF MAJOR IMPLANT-RELATED COMPLICATIONS
}

\author{
M. PANDE and B. A. NOBLE \\ Leeds
}

\begin{abstract}
SUMMARY
This study evaluates the role of intraocular lens exchange procedures in the management of major implant-related complications. Medical records of 30 patients undergoing intraocular lens exchange at the Leeds General Infirmary from 1 January 1984 to 30 November 1991 were reviewed. Details of their primary implantation surgery, intervening ophthalmic history, lens exchange surgery and outcome were analysed. Follow-up ranged from 3 to 210 weeks. Seventy-six per cent of cases achieved final visual acuity of $6 / 12$ or better. Visual acuity improved in $60 \%$, worsened in $6.6 \%$ and was unchanged in $33.4 \%$ compared with pre-operative levels. Three patients had cystoid macular oedema, 3 ocular hypertension, 2 bullous keratopathy, 1 chronic anterior uveitis and 1 patient developed a retinal detachment. This approach to managing major implant-related complications gives good visual results. There is a significant complication rate but the risk-benefit ratio justifies the use of the technique.
\end{abstract}

Intraocular lens implantation has established itself as one of the most effective and safe surgical procedures. Improvements in implant design and manufacture and in microsurgical techniques have consistently reduced the incidence and severity of major sight-threatening implantrelated complications over the years. However, infrequent major implant-related complications which require explantation do occur. The symptoms of these complications can be very distressing and an angry red painful eye is not uncommon.

After explantation aphakic glasses or contact lenses are unsatisfactory, as the patient initially warranted and expected the visual benefits of intraocular lens implantation. A replacement implant can be used in either a onestage intraocular lens exchange procedure or a two-stage secondary implant procedure. We reviewed the efficacy

Correspondence to: B. A. Noble, FRCS, FCOphth, Consultant Ophthalmologist, The General Infirmary at Leeds, Great George Street, Leeds LS1 3EX, UK. and safety of one-stage primary intraocular lens exchange surgery in 30 patients who had these procedures over a period of 8 years.

\section{SUBJECTS AND METHODS}

Medical records of all patients undergoing intraocular lens exchange at the Leeds General Infirmary from 1 January 1984 to 30 November 1991 were reviewed. Details of their primary implantation surgery, intervening ophthalmic history, and pre-operative, intra-operative and post-operative examinations relating to their intraocular lens exchange procedures were extracted, stored and analysed on a specially designed computer database.

Thirty eyes of 30 patients were identified. There were 10 male and 20 female patients. The age range was 39-92 years with a mean of 50.57 years. There were 10 right and 20 left eyes. The follow-up ranged from 3 to 210 weeks with a mean of 41 weeks; 5 cases had a follow-up of less than 16 weeks. Twenty-nine cases had a primary diagnosis of age-related cataracts while 1 case had a traumatic cataract.

The type of initial implantation surgery and the implant used in all 30 cases are shown in Table I. Six patients had complicated extracapsular cataract surgery: 1 patient (pseudoexfoliation) had a zonular dehiscence of a degree which allowed for a posterior chamber lens to be implanted, while the other 5 had posterior capsular rupture with vitreous loss and an anterior chamber lens was implanted (Choyce 1, Multiflex 4). All cases of secondary implantation had vitreous manipulation during surgery. One case had a spontaneous dislocation of a posterior chamber implant and a secondary Multiflex-type anterior chamber lens was implanted. One case had a lensectomy, sectorial iridectomy and angle recession following repair of a penetrating injury and a secondary Multiflex anterior chamber lens was implanted.

Table II shows the surgical procedures undertaken in the period between initial implantation surgery and intra- 
Table I. Initial implantation surgical procedures $(n=30)$

\begin{tabular}{|c|c|c|}
\hline Procedure & $\begin{array}{c}\text { No. of } \\
\text { cases }\end{array}$ & Type of implant \\
\hline \multicolumn{3}{|l|}{ Primary implantation } \\
\hline ICCE pupil IOL & 8 & Severin 3 , Binkhorst 3 , Bobergan 2 \\
\hline ECCE PC IOL & 10 & $\begin{array}{l}\text { Three-piece } 8 \text {, one-piece } 1 \text {, } \\
3 \mathrm{M} \text { multifocal } 1 \text {. }\end{array}$ \\
\hline \multicolumn{3}{|l|}{ ECCE PC IOL + } \\
\hline trabeculectomy & 1 & One-piece \\
\hline ECCE AC IOL & 5 & Choyce 1, Multiflex 4 \\
\hline ECCE pupil IOL & 1 & Severin \\
\hline \multicolumn{3}{|l|}{ Secondary implantation } \\
\hline $\mathrm{ICCE} 2^{\circ} \mathrm{AC} \mathrm{IOL}$ & 2 & Dubroff 1, Multiflex 1 \\
\hline ICCE $2^{\circ}$ pupil IOL & 1 & Binkhorst \\
\hline Lensectomy $2^{\circ} \mathrm{AC} \mathrm{IOL}$ & 1 & Multiflex \\
\hline ECCE AC $\mathrm{IOL}^{\mathrm{a}}$ & 1 & Multiflex \\
\hline
\end{tabular}

ICCE, intracapsular cataract extraction; IOL, intraocular lens; ECCE, extracapsular cataract extraction; $\mathrm{PC}$, posterior chamber; $\mathrm{AC}$, anterior chamber.

${ }^{\mathrm{a} S}$ Spontaneous PC IOL dislocation into vitreous.

ocular lens exchange surgery. The interval between initial implantation and lens exchange surgery ranged from 3 days to 8 years.

The patients could be categorised into three groups based on the type of initial intraocular lens implanted, as shown in Table III. The indications (more than one possible for each case) for intraocular lens exchange in the three groups are shown in Table IV.

The surgical technique consisted of horizontal whiteto-white measurement with calipers prior to a corneal section, injection of viscoelastic, intraocular lens explantation, closed chamber ocutome anterior vitrectomy (where necessary), replacement intraocular lens implantation, and closure of the section. A subconjunctival injection of betnesol and gentamicin was given at the end of surgery. Post-operatively topical steroids were used for 8-12 weeks. There were variations in the surgical technique depending on the type of initial and replacement intraocular lens, the presence of adhesions between the initial implant and ocular structures, the presence of soft lens matter and/or capsular remnants.

All pupillary implants were explanted by grasping the haptics and then carefully cutting them free of any pupillary or vitreous adhesions. Anterior chamber lens explantation was particularly difficult. The holes in the tips of the Multiflex implant haptics were associated with ring adhesions in the angle (Fig. 1). Division of these adhesions needed scissors or the tip of a needle. Pulling on the implant prior to this can result in peripheral iridodialysis

Table II. Surgical procedures in the period between initial implant surgery and exchange implant surgery $(n=3)$

\begin{tabular}{lcl}
\hline Surgical procedure & $\begin{array}{c}\text { No. of } \\
\text { cases }\end{array}$ & Initial surgery \\
\hline $\begin{array}{l}\text { McCannell suture and } \\
\text { posterior keratoplasty }\end{array}$ & 1 & ICCE pupil IOL \\
$\begin{array}{l}\text { Reposition PC IOL } \\
\text { Reposition pupil IOL }\end{array}$ & 1 & ECCE PC IOL \\
\hline
\end{tabular}

Abbreviations as in Table I.
Table III. Primary IOL types used $(n=30)$

\begin{tabular}{lc}
\hline Type of IOL & No. of cases \\
\hline Pupil supported IOL & 10 \\
Angle supported IOL & 9 \\
Posterior chamber IOL & 11 \\
\hline
\end{tabular}

IOL, intraocular lens.

and haemorrhage into the angle. The Choyce and the Dubroff lenses were more difficult to explant than the Multiflex lenses due to more extensive angle adhesions. Posterior chamber implants were explanted by dialling them. The three-piece Harris implant (which has a closed lower loop), however, had its haptics cut before explantation was possible. The lens exchange operation details, with the variations in technique used in the three groups, are shown in Table V. There were 5 cases of a penetrating keratoplasty combined with intraocular lens exchange, 4 of which had had an initial pupillary implant while 1 had had a Choyce anterior chamber implant. Trabeculectomy was combined with intraocular lens exchange in a patient who had a high intraocular pressure pre-operatively and was a known steroid responder.

Table VI shows the replacement intraocular lenses used in all cases. The choice of lens was based on the indication for exchange and the integrity of the posterior capsularzonular complex assessed at surgery. If the posterior capsular-zonular complex provided a safe support, the whole area behind the iris was checked, freed of adhesions and a replacement one-piece posterior chamber implant used. If the above criteria were not met a Multiflex anterior chamber implant with four-point fixation was used. One patient who had intracapsular surgery and a very unstable pupillary implant had a replacement one-piece posterior cham-

Table IV. Indications for IOL exchange

\begin{tabular}{|c|c|c|}
\hline Indication & $\begin{array}{l}\text { No. of } \\
\text { cases }\end{array}$ & \\
\hline \multicolumn{3}{|l|}{ Pupil supported IOL } \\
\hline Bullous keratopathy & 4 & \\
\hline Dislocated IOL & 3 & (traumatic 1 , spontaneous 2 ) \\
\hline Unstable IOL & 1 & (3 previous dislocations) \\
\hline Mobile IOL, painful eye & 1 & \\
\hline Anisometropia & 1 & \\
\hline \multicolumn{3}{|l|}{ Angle supported IOL } \\
\hline Small size for eye & 6 & (Multiflex IOL 4, Choyce IOL 1) \\
\hline UGH syndrome & 2 & (Choyce IOL 1, three-loop IOL 1) \\
\hline Bullous keratopathy & 1 & (Choyce IOL) \\
\hline Reversed oriented IOL & 1 & (Multiflex IOL) \\
\hline IOL haptic in section & 1 & (Multiflex IOL) \\
\hline Tilted IOL & 1 & (Choyce IOL) \\
\hline Dislocated IOL & 1 & (Multiflex IOL) \\
\hline Vitreous incarceration & 1 & (Multiflex IOL) \\
\hline Myopic surprise & 1 & (Multiflex IOL) \\
\hline \multicolumn{3}{|l|}{ Posterior chamber IOL } \\
\hline Sunset decentration & 2 & (three-piece IOL) \\
\hline Gross decentration & 5 & (three-piece IOL) \\
\hline Large refractive surprises & 2 & (one-piece IOL) \\
\hline Ring images, poor vision & 1 & (Multifocal) \\
\hline UGH syndrome & 1 & (Harris PC IOL) \\
\hline
\end{tabular}

IOL, intraocular lens. 


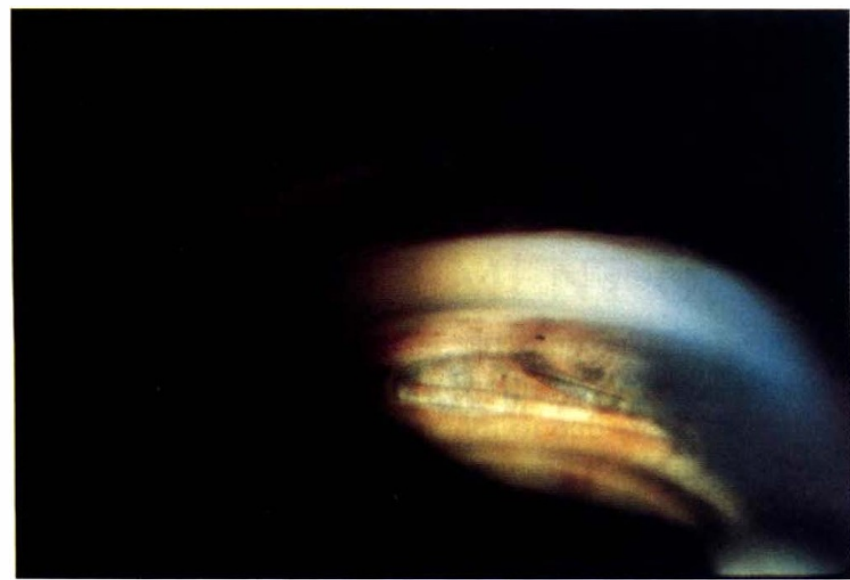

Fig. 1. Gonioscopic photograph showing peripheral iris ring synaechiae through the loop in the haptic of an anterior chamber implant.

ber implant supported by scleral sutures. Of the 5 patients who had penetrating keratoplasty combined with lens exchange, 4 had Multiflex anterior chamber replacement implants and 1 had a one-piece posterior chamber implant.

\section{RESULTS}

\section{Visual Acuity}

Pre-operative visual acuities are plotted against the final corrected post-operative visual acuity in Fig. 2. Visual acuity improved in $60 \%$ of patients and remained unchanged in $33.4 \%$ compared with pre-operative levels. A negative change from the pre-operative visual acuity was seen in only $2(6.6 \%)$ patients. One patient developed bullous keratopathy and awaits a corneal graft and the other developed a thickened posterior capsule. The patient did not attend for YAG laser capsulotomy. Final best corrected visual acuity was better than $6 / 12$ in $76.66 \%$ of

Table V. Exchange operation details

\begin{tabular}{ll}
\hline Surgical manipulations & $\begin{array}{c}\text { No. of } \\
\text { cases }\end{array}$ \\
\hline Primary pupil supported IOL & 8 \\
Pupil IOL to AC IOL & 2 \\
Pupil IOL to PC IOL & 4 \\
Penetrating keratoplasty & 6 \\
Synaechiotomy & 7 \\
Anterior vitrectomy & \\
Primary angle supported IOL & 9 \\
AC IOL to AC IOL & 1 \\
Penetrating keratoplasty & 4 \\
Synaechiotomy & 3 \\
Anterior vitrectomy & 2 \\
Lens matter removal & 2 \\
Capsular remnants removal & \\
& \\
Primary posterior chamber IOL & 6 \\
PC IOL to PC IOL & 5 \\
PC IOL to AC IOL & 1 \\
Trabeculectomy & 8 \\
Synaechiotomy & 4 \\
Anterior vitrectomy & 5 \\
Peripheral iridectomy &
\end{tabular}

IOL, intraocular lens; AC, anterior chamber; PC, posterior chamber.
Table VI. Replacement IOLs used $(n=30)$

\begin{tabular}{lc}
\hline IOL type & No. of cases \\
\hline One-piece PC IOL & 8 \\
Multiflex AC IOL & 22 \\
\hline
\end{tabular}

$\mathrm{IOL}$, intraocular lens; AC, anterior chamber; $\mathrm{PC}$, posterior chamber.

patients. The causes for a final visual acuity worse than $6 / 12$ are shown in Table VII.

\section{Complications}

Table VIII shows all the complications observed in the three groups.

Cystoid Macular Oedema (CMO). Three cases (10\%) had CMO post-operatively, 1 from each group. One of these was known to have CMO pre-operatively (UGH syndrome). None of these patients had a final post-operative visual acuity worse than their pre-operative visual acuity. All needed an anterior vitrectomy as part of their surgery. None had uveitis, glaucoma or vitreous incarceration at their final post-operative visit.

Corneal Decompensation. Corneal decompensation developed in 2 patients $(6.6 \%)$ during the early postoperative period, both of whom are awaiting corneal grafts.

Glaucoma. Pre-operatively 5 patients had ocular hypertension, 3 had the UGH syndrome, 1 was a steroid responder who had sunset decentration and 1 had a traumatic pupillary implant dislocation. The steroid responder had a trabeculectomy combined with the exchange operation and was normotensive post-operatively. Two of the patients with the UGH syndrome were normotensive postoperatively with just the exchange operation, while 1 needed a trabeculectomy 12 weeks after the exchange operation. The patient with the traumatic implant dislocation was normotensive post-operatively on topical beta blocker therapy.

Two patients who were normotensive pre-operatively developed ocular hypertension post-operatively. In 1 case treatment was not required after 12 weeks. The other patient was controlled successfully on topical beta blocker therapy.

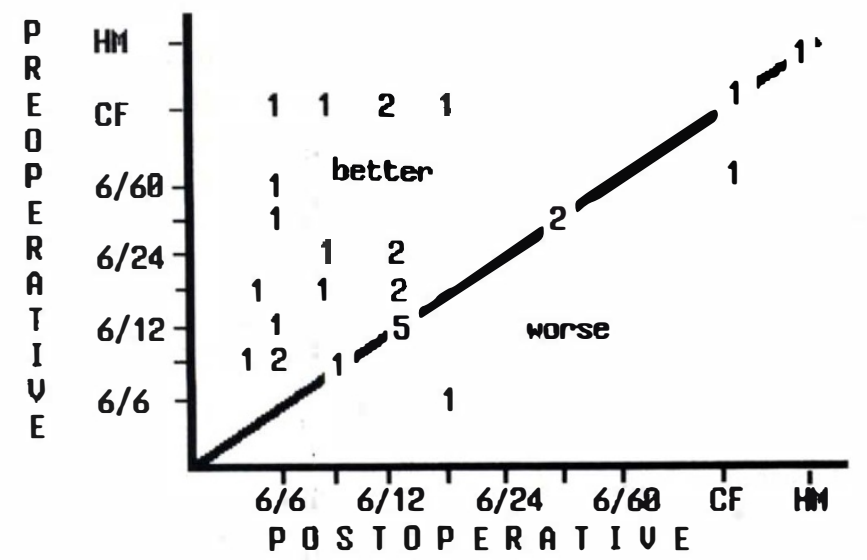

Fig. 2. Comparison of pre-operative and final corrected postoperative visual acuities for all patients. 
Table VII. Causes of best corrected final visual acuity (VA) worse than $6 / 12$

\begin{tabular}{lcl}
\hline & Final & \\
Initial implant group & VA & Cause \\
\hline Pupil & $6 / 18$ & Cystoid macular oedema \\
& HM & Bullous keratopathy \\
& CF & Bullous kerałopathy \\
Anterior chamber & $6 / 24$ & Capsular thickening \\
& CF & Cystoid macular oedema \\
Posterior chamber & $6 / 36$ & Diabetic maculopathy \\
\hline
\end{tabular}

$\mathrm{HM}$, hand movement; $\mathrm{CF}$, counting fingers.

Chronic Anterior Uveitis. Uveitis lasting longer than 8 weeks post-operatively was seen in only 1 patient. This patient is on long-term topical steroid therapy.

Peaked Pupil. A peaked pupil due to an iris tuck developed in 1 case of an anterior chamber to anterior chamber implantexchange. The visual acuity in this patient was $6 / 9$ and no further action was deemed necessary.

Retinal Detachment. One patient developed a retinal detachment which was successfully reattached surgically.

\section{DISCUSSION}

The distribution of the explanted primary implant type (pupil supported, anterior chamber intraocular lens, posterior chamber intraocular lens) was roughly even. This sample distribution differs from that reported by various authors ${ }^{1-4}$ who found anterior chamber lenses to be the commonest type of implant requiring explantation. This difference reflects the practice styles in the catchment area of Leeds General Infirmary.

Of the exchanged implants $33.33 \%$ (10 of 30 ) were pupil supported. Pseudophakic bullous keratopathy (Fig. 3) and implant dislocation were the commonest indications for lens exchange surgery in this group. This finding is consistent with previous reports ${ }^{2.3}$ and highlights the flawed concept of pupil supported implants.

Anterior chamber implants formed 30\% (9 of 30) of the implants exchanged in this series. Seven of the 9 were Multiflex-type implants with four-point fixation; there was also 1 Choyce and 1 Dubroff implant. The commonest indication for surgery in this group was a small-sized implant (Fig. 4). The UGH syndrome was seen in the 2 cases with the Choyce and Dubroff implants. Multiflextype implants have been shown to be safe and effective as secondary implants ${ }^{5}$ and as a backup implant in extra-

Table VIII. Complications of lens exchange surgery $(n=30)$

\begin{tabular}{lccc}
\hline & \multicolumn{3}{c}{ Initial implant group } \\
\cline { 2 - 4 } Complication & Pupil IOL & AC IOL & PC IOL \\
\hline Cystoid macular oedema & 1 & 1 & 1 \\
Bullous keratopathy & 2 & 0 & 0 \\
Glaucoma & 2 & 1 & 0 \\
Chronic uveitis & 1 & 0 & 0 \\
Peaked pupil (iris tuck) & 0 & 1 & 0 \\
Retinal detachment & 0 & 1 & 0 \\
\hline
\end{tabular}

IOL, intraocular lens; $\mathrm{AC}$, anterior chamber; $\mathrm{PC}$, posterior chamber.

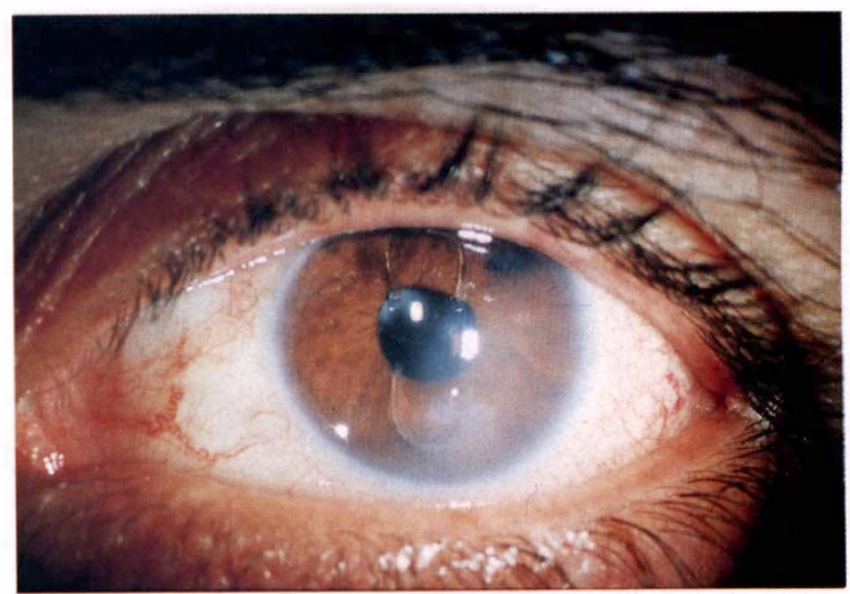

Fig. 3. Pupil implant exchange indication: pseudophakic bullous keratopathy.

capsular surgery. ${ }^{6}$ Five of the 7 cases with primary Multiflex implants had complicated extracapsular surgery. The number of cases with implants that were small for the eye highlights the importance of measuring the horizontal white-to-white diameter prior to vitrectomy, which reduces the ocular volume intra-operatively. Choyce and Dubroff implants are not in common use at present. UGH syndrome has been previously reported with these implants, ${ }^{7}$ as it has with the closed loop anterior chamber implants. ${ }^{8}$

Posterior chamber implants formed $36.6 \%$ (11 of 30) of the implants exchanged in this series. Problems with decentration (Fig. 5) were the commonest indications for lens exchange in this group of patients, which is consistent with other reports. ${ }^{1,2}$ Posterior chamber implant decentration is a multifactorial problem, with factors relating to haptic flexibility, implant fixation ${ }^{9}$ and capsulotomy technique all playing a part. ${ }^{10}$ All the posterior chamber lenses exchanged for decentration were three-piece implants with polypropylene haptics. This suggests that the more flexible polypropylene haptics were an important factor in implant decentration in our cases. There were 2 patients who had a one-piece PMMA posterior chamber lens; the indication in both these cases was a large refractive surprise due to errors in pre-operative implant power cal-

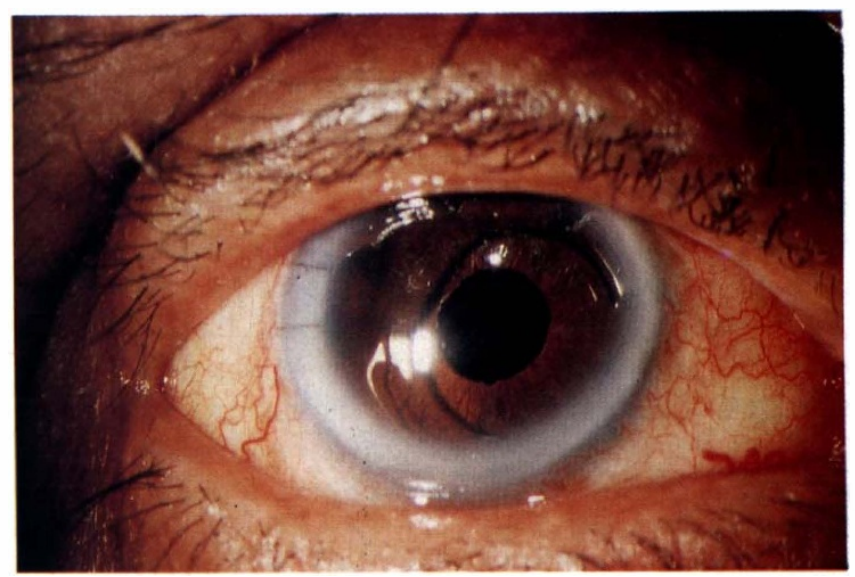

Fig. 4. Anterior chamber implant exchange indication: smallsized Multiflex implant. 


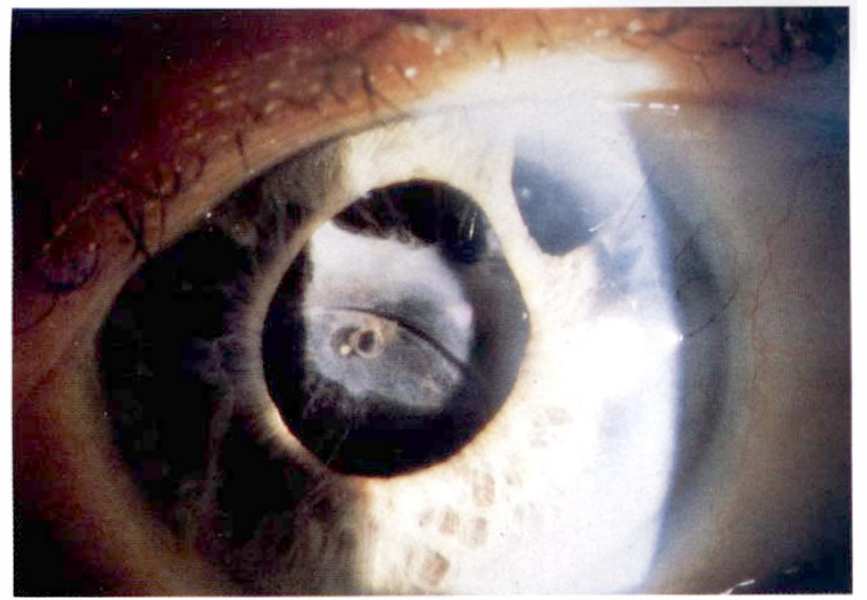

Fig. 5. Posterior chamber implant exchange indication: sunset decentration.

culations. There was 1 case of a 3M Multifocal implant in this group. This patient had ring images, pigment chaffing and diminished visual acuity. The ring images were thought to be related to the implant design based on diffractive optics.

Vitreous manipulation, which is associated with increased complications, ${ }^{11}$ was most commonly required in the pupil supported implant group. This is related to the primary cataract extraction being an intracapsular extraction in these cases. Vitrectomy was also required in patients who had complicated extracapsular surgery, indicating the suboptimal surgical management of the vitreous during primary surgery. Synaechiotomy was most difficult in the anterior chamber lens group, where the holes at the haptic tips formed ring adhesions to the angle. Penetrating keratoplasty was the commonest associated surgical procedure with lens exchange surgery. There were 5 patients who had penetrating keratoplasty combined with implant exchange surgery, 4 of whom had initial pupil supported implants which 1 had a Choyce anterior chamber implant.

Multiflex-type one-piece all-PMMA implants with four-point fixation were the commonest exchange implants (22 of 30 ) used in this series. These implants require a correct choice of size and an optimal implantation technique. Provided these conditions are met they give good visual results and are safe. This is further supported by the good results seen in the patients who had complicated extracapsular surgery and 'small for eye' Multiflex implants exchanged for Multiflex implants of the correct size. Eight cases had one-piece PMMA posterior chamber exchange implants. It is important to assess whether the posterior capsular-zonular complex provides adequate support for implantation, as well as to clear the retroiridial space of any adhesions. Only 1 patient had a posterior chamber exchange implant supported by scleral sutures. The early results of this technique are promising ${ }^{12}$ but long-term follow-up is necessary to establish the advantage of this technique over that of implanting a Multiflex anterior chamber implant in cases without adequate posterior capsular support. A final corrected visual acuity better than or equal to $6 / 12$ was achieved by $76.66 \%$ of patients in this series. Compared with their pre- operative visual acuity $60 \%$ showed an improvement, $33.4 \%$ had no change and only $6.6 \%$ ( 2 of 30 ) had worsened. The drop in final visual acuity from the preoperative level was due to bullous keratopathy in 1 case (awaiting penetrating keratoplasty) and a thickened posterior capsule in the other. This latter patient did not attend for a YAG laser capsulotomy and was lost to further follow-up. Other studies ${ }^{2,13-15}$ have also reported good visual results with implant exchange procedures, with visual acuities improving or remaining unchanged from their pre-operative level in about $83-90 \%$ of cases.

Cystoid macular oedema (10\%) and glaucoma (10\%) were the commonest complications of lens exchange surgery, followed by bullous keratopathy $(6.6 \%)$. Retinal detachment, chronic uveitis and peaked pupil occurred in 1 case each. Brown and Snead ${ }^{13}$ also found CMO to be the commonest cause of decreased vision in patients who had lens exchange surgery.

The complications were most commonly seen in the pupil supported implant group. This could be a reflection of the more serious nature of the indications for lens exchange surgery seen with these implants, namely pseudophakic bullous keratopathy. Sinskey et al. ${ }^{14}$ in their lens exchange series have also reported on the poorer prognosis in patients who had pupil supported implants.

The majority of complications associated with intraocular lens implant surgery can be managed conservatively. There are, however, some complications which require the offending implant to be explanted. Intraocular lens explantation is an additional surgical trauma and should be embarked upon only if the pre-operative assessment indicates that the patient's condition can be improved or stabilised by the procedure. Alpar ${ }^{16}$ initially proposed several indications for intraocular lens explantation. These have been modified in recent times with the advent of viscoelastic materials and improved microsurgical techniques. The decreasing use of pupil and anterior chamber implants and more widespread use of posterior chamber implants will further modify the indications for intraocular lens explantation/exchange procedures. Decentration is likely to be the most common indication for such procedures in the future. Further, if implant exchange is considered in such patients it is imperative to assess whether the tissue damage caused by the complicating implant will allow for safe implantation of an exchange implant. The choice of an exchange implant type has to be based on available capsular support, iridocapsular adhesions, the state of the vitreous and the peripheral iridocorneal anatomy. The results justify the use of implant exchange surgery in the management of a variety of complications; however, the decision to embark on lens exchange surgery has to be taken after individual assessment of the patient's condition based on the factors outlined above.

Key words: Implant complications, Intraocular lens exchange.

\section{REFERENCES}

1. Solomon KD, Apple DJ, Mamalis N, et al. Complications of 
intraocular lenses with special reference to an analysis of 2,500 explanted intraocular lenses. Eur J Implant Refract Surg 1991;3:195-200.

2. Mamalis N, Crandall AS, Pulsipher MW, et al. Intraocular lens explantation and exchange. J Cataract Refract Surg 1991;17:811-18.

3. Kraff MC, Sanders DR, Raanan MG. A survey of intraocular lens explantations. J Cataract Refract Surg 1986;12:644-50.

4. Doren GS, Stern GA, Driebe WT. Indications for and results of intraocular lens explantation. J Cataract Refract Surg 1992;18:79-85.

5. Hayward JM, Noble BA, George N. Secondary intraocular lens implantation: eight years' experience. Eye 1990;4: 548-56.

6. Apple DJ et al. Anterior chamber lenses, part I, complications and pathology and a review of designs. J Cataract Refract Surg 1987; 13:157-74.

7. Ellingson FT. Complications with the Choyce mark VIII anterior chamber lens implant (uveitis, glaucoma, hyphaema). J Am Intraocul Implant Soc 1977;3:199-201.

8. Hagan JC. Additional long-term follow-up on the IOLAB Azar 91-Z anterior chamber intraocular lens. J Cataract Refract Surg 1986;12:398-400.
9. Hansen SO, Tetz MR, Solomon KD, et al. Decentration of flexible loop posterior chamber intraocular lenses in a series of 222 post-mortem eyes. Ophthalmology 1988;95:344-49.

10. Gimbel HV, Neuhann T. Development, advantages and methods of the continuous circular capsulorhexis technique. J Cataract Refract Surg 1990;16:31-37.

11. Vail D. After-results of vitreous loss. Am J Ophthalmol 1965;59:573-86.

12. Nabors G, Varley MP, Charles S. Ciliary sulcus suturing of a posterior chamber intraocular lens. Ophthalmic Surg 1990; 21:263-5.

13. Brown DC, Snead JW. Intraocular lens implant exchanges. J Am Intraocular Implant Soc 1985;11:376-9.

14. Sinskey RM, Garwood JL, Patel J. Intraocular lens exchange: a five-year experience. Cataract 1985;2(7): 12-18.

15. Koenig SB, McDermott ML, Hyndiuk RA. Penetrating keratoplasty and intraocular lens exchange for pseudophakic bullous keratopathy associated with a closed loop anterior chamber intraocular lens. Am J Ophthalmol 1989;108:43-8.

16. Alpar JJ. Removal of intraocular lenses: explantation and/or lens exchange. Ann Ophthalmol 1987;19:194-8. 